\title{
Realizing Broadbands of Strong Nonlinear Coupling in Nanoelectromechanical Electron Shuttles
}

\author{
Chulki Kim, ${ }^{1}$ Marta Prada, ${ }^{2,3,4, *}$ Gloria Platero, ${ }^{3}$ and Robert H. Blick ${ }^{4,5,6}$ \\ ${ }^{1}$ Sensor System Research Center, Korea Institute of Science and Technology, Seoul 136-791, Republic of Korea \\ ${ }^{2}$ I. Institut für Theoretische Physik, Universität Hamburg, Jungiusstrasse 9, 20355 Hamburg, Germany \\ ${ }^{3}$ Instituto de Ciencias Materiales de Madrid, ICMM-CSIC, Sor Juana Ines de la Cruz 3, 28049 Madrid, Spain \\ ${ }^{4}$ Department of Physics, University of Wisconsin-Madison, 1150 University Avenue, Madison, Wisconsin 53706, USA \\ ${ }^{5}$ Institut für Angewandte Physik, Universität Hamburg, Jungiusstr. 11, 20355 Hamburg, Germany \\ ${ }^{6}$ Department of Electrical and Computer Engineering, University of Wisconsin-Madison, \\ 1415 Engineering Dr. Madison, Wisconsin 53706, USA
}

(Received 28 February 2013; revised manuscript received 1 June 2013; published 5 November 2013)

\begin{abstract}
We demonstrate the transition of a coupled electron shuttle from a stable to a strongly nonlinear response at room temperature. Within this transition we observe the coupled shuttle's response to change from Coulomb controlled to conventional field emission. This parametric process is fully reversible and occurs within a broad frequency range. In combination, the large current and wide frequency band enable energy harvesting applications. The experimental data and the numerical calculations both indicate that the source of the nonlinearity is given by the electromechanical coupling of electron shuttling.
\end{abstract}

DOI: 10.1103/PhysRevLett.111.197202

PACS numbers: 85.85.+j, 05.45.Xt, 47.20.Ky, 81.07.Oj

Vibration energy harvesting is attracting considerable attention, in devices where replacement of exhausted batteries is not an option. A crucial limitation of resonant vibration energy harvesters is that the best performance of the devices is limited to a very narrow bandwidth around the fundamental resonance frequency. If the excitation frequency deviates slightly from the resonance condition, the power output is drastically reduced, preventing most cantilever-based energy harvesters from practical applications [1]. This limitation has motivated extensive research in microelectromechanical and nanoelectromechanical systems (NEMS), where nonlinear, bistable oscillators are believed to provide the best performance [2]. In particular, arrays of nanopillars are being extensively studied for their potential applications as energy harvesters [3] apart from conventional solar cells ideas [4].

In this Letter, we investigate the most fundamental component for the realization of bistable oscillators: a coupled pendula, consisting of metallic islands on top of two silicon nanopillars. Such structures form mechanical resonators-also coined electron shuttles. Since the original suggestion of the shuttle phenomenon [5], there has been increasing interest due to the rich new physics phenomena as the electronic and mechanical degrees of freedom combine [6-9]. Electron shuttles are leading candidates to many functionalities in future industry, as they operate at radio frequencies ( $\mathrm{rf}$ ), possess a set of resonance frequencies, and reveal Coulomb blockade even at room temperature [10]. Spontaneous symmetry breaking has also been observed in these systems [11], revealing Arnol'd tongues in the frequency domain [12].

Here, we focus on weak rf excitation of a coupled nanopillar shuttle. We show that the coupled oscillators can be parametrically driven into a highly nonlinear regime greatly affecting the motion of the shuttle. In this nonlinear regime, field emission amplifies the response by several orders of magnitude. Such a large current then has the potential to form the basis for energy harvesting via nanomechanical shuttles. The effect is observed over an extremely broadband of excitation frequencies, highly desirable for energy harvesters [13]. Moreover, the system presents the great advantage of being operated at room temperature and is scalable, in contrast to other NEMS-based approaches [14].

A scanning electron microscope image of a coupled nanomechanical pendula is shown in Fig. 1(a): the diameters of the gold islands are around 50 and $64 \mathrm{~nm}$, respectively. They are placed on top of the nanopillars etched out of a silicon-on-insulator substrate, with a top silicon layer of $190 \mathrm{~nm}$ thinness and an insulating layer of $350 \mathrm{~nm}$. (a)

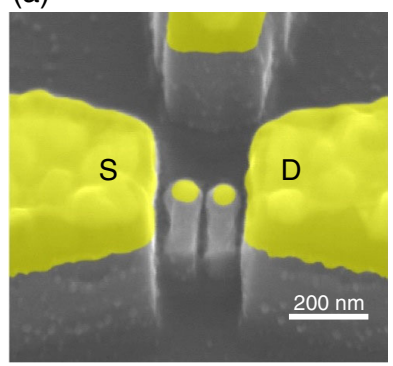

(b)

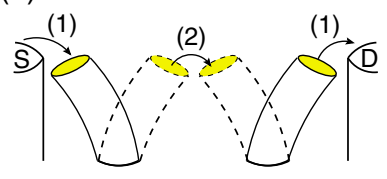

(c)

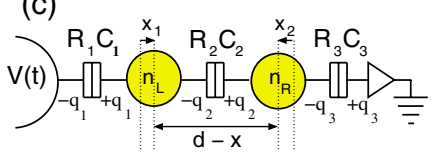

FIG. 1 (color online). (a) Two coupled nanomechanical shuttles: the metallic islands on top provide the conduction path for the electrons between source $S$ and drain $D$ contacts. (b) The flexural modes drive a mechanically assisted direct current-the shuttling current-where the center of mass remains at rest. (c) Circuit diagram with the mechanically tunable tunneling barriers between the leads and electron islands (see text for details). 
The $50 \mathrm{~nm}$ top gold layer serves as the electrical conduction path as well as an etch mask in the dry etch step to mill out the surrounding silicon-on-insulator material. To ensure electron transport only via the metallic islands, the substrate is etched down to the insulating layer. A schematic circuit diagram of the measurement setup is shown in Fig. 1(c). The three junctions are characterized by a resistance $R_{j}(x), j=1,2,3$, where $x=x_{1}-x_{2}$ accounts for the relative displacement of the islands.

All measurements are performed under vacuum ( $p \sim 10^{-5}$ mbar) at room temperature. External rf signals are applied via impedance matched transmission lines in order to minimize signal losses along the line. The dc output current reveals the resonance spectrum as expected and demonstrated before [11], where dynamical symmetry breaking causes parametric instability [15]. The low frequency regime containing the enhanced current response is shown in Fig. 2(a) at three different power levels. Peaks rise when increasing the rf power level from 0 to $15 \mathrm{dBm}$. The current-voltage characteristics at three frequenciesat $68 \mathrm{MHz}$ (square), $72 \mathrm{MHz}$ (dot), and $92 \mathrm{MHz}$ (triangle) - are shown in Fig. 2(b). The inset of Fig. 2(b) gives the average number of electrons transferred per cycle in the low bias regime $\left(V_{\mathrm{ac}} \leq 0.45 \mathrm{~V}\right)$, where a steplike structure due to Coulomb blockade is apparent.

As a first step, we want to model Coulomb blockade. This also serves as a test for our modeling of the nonlinear properties of the circuit. For this, we focus on the tunneling process across junction "1" [see Fig. 1(c)]. The energy an electron needs to overcome reads [16]

$$
\mu_{1}^{\rightleftarrows}\left(x, n_{L}, V\right) \simeq \frac{e^{2}}{C_{\alpha_{1}}(x)}\left[1 \mp 2 n_{L} \pm \frac{V / V_{\mathrm{th}}^{1}}{1-x / d}\right],
$$

where $V$ is the bias voltage, $C_{\alpha_{1}}$ is a capacitance constant determined by the geometry of the system, $d \simeq 15 \mathrm{~nm}$ is the interdot distance, with $|x / d|<1 / 2, n_{L}$ is the number of excess electrons in the left island, and the arrow labels the direction of the movement of the electron. Here, we have defined the static $(x=0)$ threshold voltage, $V_{\text {th }}^{1}$. The criterion for a tunneling event to occur is that the
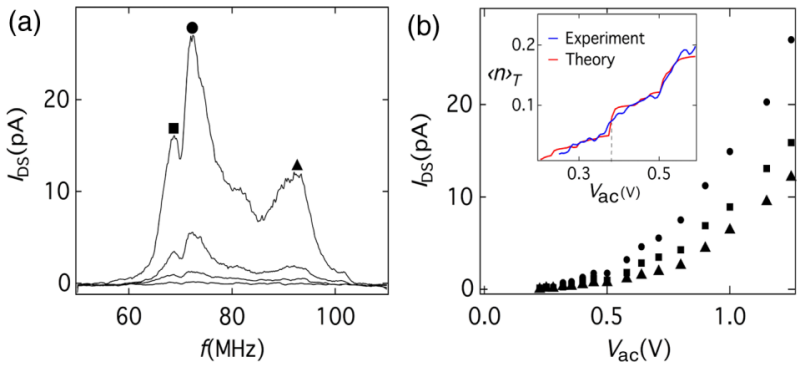

FIG. 2 (color online). (a) Output current with external rf signal of $0,5,10$, and $15 \mathrm{dBm}$. (b) $I V$ characteristics at three different frequencies $68 \mathrm{MHz}$ (square), $72 \mathrm{MHz}$ (dot), and $92 \mathrm{MHz}$ (triangle). The inset shows a steplike current in the low bias limit due to the mechanically enhanced Coulomb blockade (see text). free energy is lowered after the process, $\mu_{1}^{\rightleftarrows}<0$. If the magnitude of the voltage is just below the threshold, say, $|V|=V_{\mathrm{th}^{1}}-\varepsilon \quad$ with $\varepsilon>0$, then $\mu_{1}<0 \quad\left(\mu_{1}^{\leftarrow}<0\right)$ requires $V<0(V>0)$ and $x>\varepsilon / V_{\mathrm{th}^{1}}$; i.e., tunneling is only possible when the island separates from the electrode $(x>0)$. Basically, a decrease in the Coulomb blockade threshold occurs as the islands move away from the electrodes, due to the mutual capacitance dependence on relative distance [17]. However, the tunneling process is exponentially suppressed as the tunneling distance increases.

On the contrary, just above the threshold $|V|=V_{\mathrm{th}^{1}}+\varepsilon$, tunneling processes become energetically favorable, as $\mu_{1}^{\rightleftarrows}<0$ even at $x<0$, with a consequent enhancement of the tunneling current. Due to the integer nature of the charge, a steplike current is to be expected as a function of the voltage in the shuttling regime [5]. The blue curve of the inset of Fig. 2(b) shows the average number of transferred electrons per cycle, $\langle n\rangle_{T}$ as a function of $V_{\mathrm{ac}}$, where current steps are apparent. The red curve is a theoretical fit, obtained by solving the master equation self-consistently with the mechanical equation [18]. We note an overall linear increase of the current with $V_{\text {ac }}$, due to thermal broadening.

We now superimpose a dc bias voltage $V_{\mathrm{dc}}=-0.1 \mathrm{~V}$ on the rf signals using a bias-tee: $V(t)=V_{0}[\sin (f t)+\beta]$. An anomalously large nonlinear response is observed at a low rf level $(\sim 3-5 \mathrm{dBm})$. Figure $3(\mathrm{a})$ shows stroboscopic traces at 3.1, 4, and $5.4 \mathrm{dBm}$. Dramatic changes in the response are apparent [note the logarithmic scale in Fig. 3(a)], expressed by extremely large field emission currents. In this region, mechanically assisted self-enhanced oscillations appear as a consequence of the parametric instability induced by a pitchfork bifurcation.

A subsequent drastic enhancement of the direct current occurs-key for energy harvesting-when the FowlerNordheim tunneling limit is reached [19]. This results in a current 2 orders of magnitude larger than the one observed in the Arnol'd tongues. Figure 3(b) shows the high-power rf regime, where Arnol'd tongues are visible at a signal power of $\sim 10-15 \mathrm{dBm}$. This is similar to the case with only the rf signal applied, see Fig. 2(a). The whole structure in the frequency domain is summarized in the color scale in Fig. 3(c). Note that the color scale is linear up to $10 \mathrm{pA}$ and logarithmic thereafter.

Apart from strongly enhanced current levels, we can also state that the resonance width or active bandwidth (BW) for energy harvesting is increased by roughly 1 order of magnitude. This is the second key ingredient for harvesting applications. Comparing Figs. 3(a) and 3(b), the linear response's BW at about $75 \mathrm{MHz}$ is a bit above $5 \mathrm{MHz}$, while the nonlinear response's BW spans the entire data range shown of $50 \mathrm{MHz}$. Hence, entering the nonlinear regime of the coupled electromechanical shuttles truly improves the BW. 

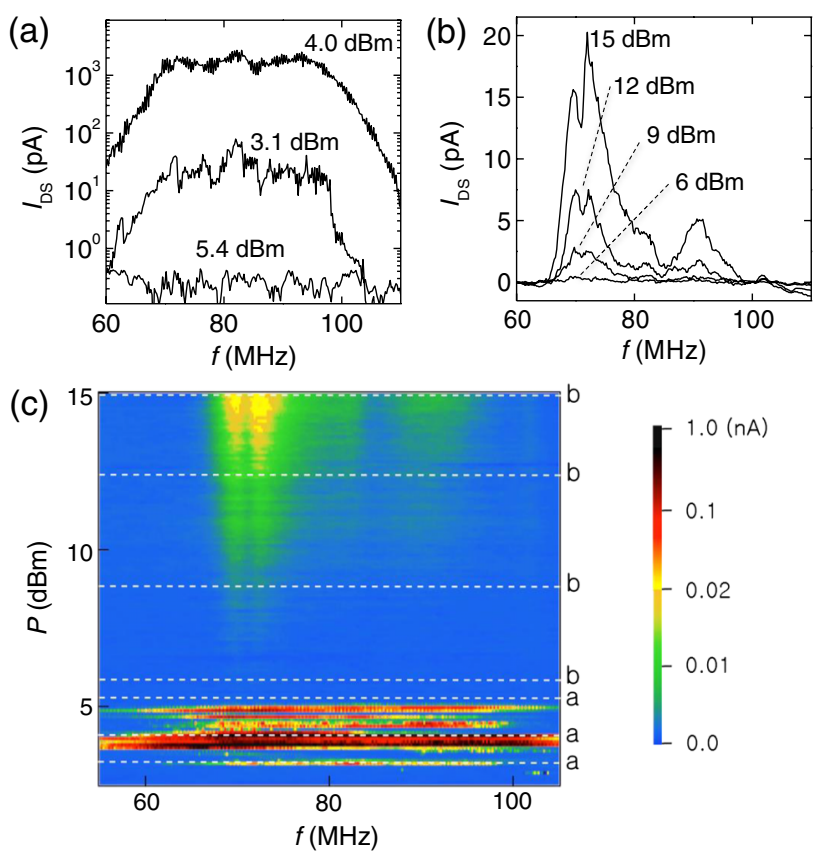

FIG. 3 (color online). Output direct currents with different external rf signal powers: (a) 3.1, 4, and $5.4 \mathrm{dBm}$ superimposed on a bias voltage of $-100 \mathrm{mV}$. (b) The conventional periodic responses are recovered above a certain rf signal power level of $\sim 6 \mathrm{dBm}$. (c) Color-scale graph of output current via coupled nanoscale pendula in the frequency domain with continuous ac power $P$ increase. Thin dashed lines indicate the line plots shown in (a) and (b). Note that the scale is logarithmic for direct current larger than $10 \mathrm{pA}$, and linear otherwise.

In order to quantify this nonlinear behavior further, we model it in terms of mechanical instabilities. We describe the electrodynamics of the coupled shuttle with a varying relative charge $n=n_{L}-n_{R}$, subject to a force produced by the electric field $-V(t) / L$,

$$
\ddot{x}+x+\frac{\gamma}{f_{p}} \dot{x}=-\frac{e V(t) n(t, x)}{m f_{p}^{2} L \lambda},
$$

where $L$ is the source-drain distance, $x$ is conveniently expressed in units of the tunneling distance, $\lambda . \gamma$ is the dynamic damping and $f_{p}$ is the oscillator's eigenfrequency. The last term of Eq. (1) determines the response of the system, as we describe it below. In the limit of small oscillations (linear regime), the amplitude of the relative displacement of the nanoislands is comparable to the tunneling distance, $\lambda$. Moreover, in the adiabatic limit, the charge follows the excitation, and thus, multipliers can be encountered $n(t, x) \propto V(t) \phi(x)$.

Using classical electrodynamics we find $\phi(x)[15,16]$, and we express the last term of Eq. (1) with the explicit $x$ dependency,

$$
\frac{e V(t) n(t, x)}{m f_{p}^{2} L \lambda} \simeq \alpha[\sin (f t)+\beta]^{2}\left[x-\frac{2 \lambda}{d} x^{2}-\frac{3}{16} x^{3}\right],
$$

where we have defined the dimensionless parameter $\alpha=3 C V_{0}^{2} / 4 m L \lambda f_{p}^{2}$ that quantifies the strength of the $\mathrm{rf}$ excitation. $\alpha$ is the ratio of the electric $\left(\sim C V_{0}^{2} / L\right)$ and mechanical forces $\left(\sim m \lambda f_{p}^{2}\right)$. The modified Mathieu equation is then obtained within the weak electromechanical coupling limit, where $\alpha$ is arbitrarily small.

We finally solve Eq. (1) using the Poincaré-Lindstedt method within the weak electromechanical coupling limit. Evaluation routines [20] allow us to numerically find unstable regions and the associated bifurcation diagrams. Figure 4 represents such a bifurcation diagram in the parameter space spanned by $\alpha$ and $f$. Instabilities are expected in the solid regions, where self-sustained oscillations in the Fowler-Nordheim limit can occur.

The insets show, schematically, two different phase portraits associated with the solid-colored regions. In the red-colored region the origin is unstable, in the sense that even if the pillars are initially at rest, the electrostatic field creates oscillations in the stationary limit. As the red phase portraits of the insets illustrate, the associated trajectories start near the origin and converge to either of the two oscillatory stable solutions. Hence, an increase in frequency leads to two nontrivial stable nodes, which coexist in phase space. This scenario is termed a supercritical pitchfork bifurcation in the field of nonlinear dynamics, due to the semblance with the corresponding stroboscopic plot (lower right inset of Fig. 4).

In the green region, the origin becomes stable again, but two additional nontrivial stable nodes appear. This implies that only if the shuttling regime described above has been

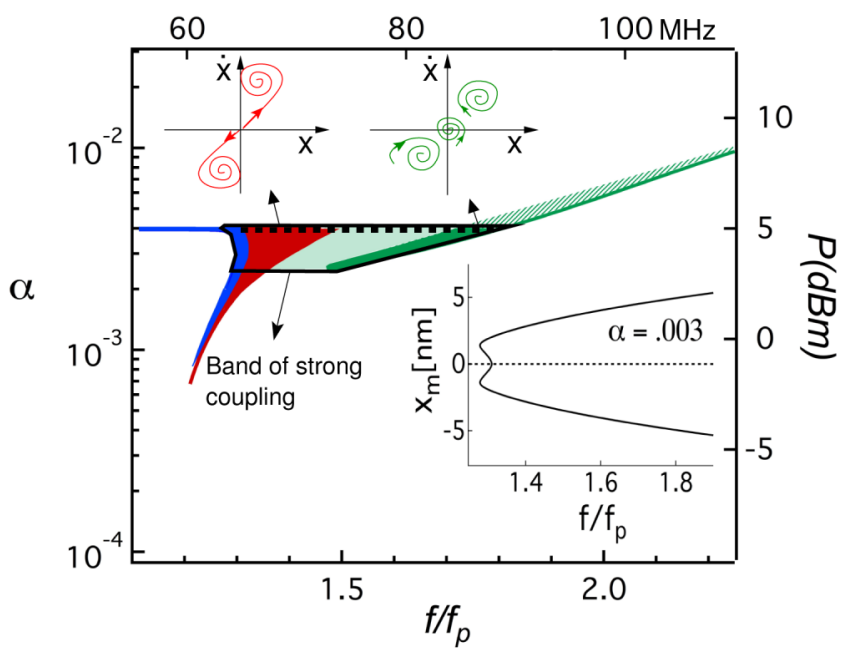

FIG. 4 (color online). Bifurcation diagram showing unstable regions where self-sustained oscillations are expected. The two upper insets show corresponding phase portraits: In the pitchfork bifurcation (red or dark gray) the origin is unstable, whereas in the green (gray) region, the attractor at the origin coexists with another two nontrivial stable nodes. The lower inset shows the amplitude $x_{m}$ of the relative deflection for the stable solution along the black broken trace, $\alpha \simeq 3 \times 10^{-3}$. 
TABLE I. Materials parameters used in this Letter. (TW = this Letter, parameters to match Fig. 3).

\begin{tabular}{lccc}
\hline \hline Parameter & Value & Units & Source \\
\hline$C$ & 4 & $\mathrm{aF}$ & {$[10]$} \\
$L$ & 150 & $\mathrm{~nm}$ & {$[10]$} \\
$m$ & $2 \times 10^{-18}$ & $\mathrm{~kg}$ & {$[10,11]$} \\
$d$ & 45 & $\mathrm{~nm}$ & {$[10]$} \\
$\lambda$ & 15 & $\mathrm{~nm}$ & $\mathrm{TW}$ \\
$\gamma$ & .05 & $f_{p}$ & $\mathrm{TW}$ \\
$f_{p}$ & 50 & $\mathrm{MHz}$ & $\mathrm{TW}$ \\
$Z$ & 50 & $\Omega$ & $\mathrm{TW}$ \\
\hline \hline
\end{tabular}

reached, the oscillations are kept throughout the light green region of Fig. 4. A band of strong nonlinear response is then observed for a wide range of frequencies.

The instability is known to be parametric, as it is caused by a smooth change in the electromechanical coupling $\alpha$. The mechanical vibrations of the nanopillars perturb the charge balance on the islands, causing accelerations that further enhance the displacements of the shuttles. Those nonlinear modes are the ones to exploit for energy harvesting.

In the shuttling regime the number of electrons is $n(t, x) \simeq n(t)$, i.e., a square wave synchronized with the excitation $V(t)[16,21,22]$. The last term of Eq. (1) would then read,

$\frac{e V(t) n(t)}{m f_{p}^{2} L \lambda} \simeq \frac{e V_{0}(\sin f t+\beta)\left(n_{\mathrm{av}}+n_{0} \cos f t+\cdots\right)}{m f_{p}^{2} L \lambda}$.

Now, solving Eq. (1) analytically, we find nontrivial stable points at any frequency $f$. In other words, once the adiabatic self-oscillatory regime is reached, the transition into the shuttling regime follows. Making use of [16], we find that a positive energy can be pumped into the system when $e V_{0} \beta n_{0} / L m f_{p}^{2} \lambda \geq \gamma x_{m}$. The Fowler-Nordheim limit will then follow, with a consequent enhancement of the response. Given the parameters of Table I, we can estimate that this occurs when $n_{0} \geq 4$.

We match the experimental results, identifying the theoretically encountered band of instability with the experimental one, as noted in the right and top axis of Fig. 4 ( $\alpha$ and $f$ have arbitrary units on the left and bottom axes). This involves the parameters given in Table I. Based on this data, we can state that an array of coupled nanopillar shuttles with attainable dimensions, i.e., diameters around $50 \mathrm{~nm}$, tuned into a slightly nonlinear state, will be an excellent nanomechanical energy harvester.

In summary, we have shown that the mechanical response of a coupled electron shuttle leads to steplike current, showing the imprints of the Coulomb blockade at room temperature. We observe parametrically driven bifurcations, where a smooth change in the electromechanical coupling leads to an oscillatory adiabatic regime, followed by a self-oscillatory scenario within the shuttling limit, and ending with a nonlinear response. The resulting field emission current is then dramatically enhanced by 2 orders of magnitude as compared to the conventionally recorded shuttling currents. In addition the effective bandwidth of the mechanical system is enhanced by 1 order of magnitude.

The authors would like to thank the Defense Advanced Research Projects Agency (DARPA) for support through the NEMS-CMOS Program (No. N66001-07-1-2046), the University of Wisconsin-Madison for support by a DraperTIF Award, the Wisconsin Alumni Research Foundation (WARF) for a Clean Tech Grant within the Accelerator Program, the Spanish Ministry of Education [Programs No. SB2009-007, No. MAT 2011-24331 and ITN, Grant No. 234970 (EU)] and the Korea Institute of Science and Technology (KIST) for support through the KIST Institutional program (2V03030) and the KIST Future Fundamental Research Program.

*mprada@physnet.uni-hamburg.de

[1] S.P. Beeby, M. J. Tudor, and N. M. White, Meas. Sci. Technol. 17, R175 (2006).

[2] F. Cottone, H. Vocca, and L. Gammaitoni, Phys. Rev. Lett. 102, 080601 (2009).

[3] J. Li, H. Yu, S. M. Wong, G. Zhang, X. Sun, P. G.-Q. Lo, and D.-L. Kwong, Appl. Phys. Lett. 95, 033102 (2009).

[4] M. Law, L. E. Greene, J. C. Johnson, R. Saykally, and P. Yang, Nat. Mater. 4, 455 (2005); M. D. Kelzenberg, D. B. Turner-Evans, B. M. Kayes, M. A. Filler, M. C. Putnam, N. S. Lewis, and H. A. Atwater, Nano Lett. 8, 710 (2008); S. M. Wong, H. Y. Yu, J. S. Li, G. Zhang, P. Lo, and D. L. Kwong, IEEE Electron Device Lett. 31, 335 (2010).

[5] L. Y. Gorelik, A. Isacsson, M. V. Voinova, B. Kasemo, R. I. Shekhter, and M. Jonson, Phys. Rev. Lett. 80, 4526 (1998).

[6] T. Novotný, A. Donarini, and A.-P. Jauho, Phys. Rev. Lett. 90, 256801 (2003).

[7] F. Pistolesi and R. Fazio, Phys. Rev. Lett. 94, 036806 (2005).

[8] N. Nishiguchi, Phys. Rev. Lett. 89, 066802 (2002).

[9] A.D. Armour and A. MacKinnon, Phys. Rev. B 66, 035333 (2002).

[10] C. Kim, M. Prada, and R. H. Blick, ACS Nano, 6, 651 (2012).

[11] C. Kim, J. Park, and R. H. Blick, Phys. Rev. Lett. 105, 067204 (2010).

[12] J. A. Glazier, M. H. Jensen, A. Libchaber, and J. Stavans, Phys. Rev. A 34, 1621 (1986).

[13] R. Masana and M. F. Daqaq, J. Appl. Phys. 111, 044501 (2012).

[14] B. Lassagne, Y. Tarakanov, J. Kinaret, D. Garcia-Sanchez, and A. Bachtold, Science 325, 1107 (2009).

[15] K. H. Ahn, H. C. Park, J. Wiersig, and H. Jongbae, Phys. Rev. Lett. 97, 216804 (2006).

[16] M. Prada and G. Platero, Phys. Rev. B 86, 165424 (2012).

[17] T. Nord, L. Y. Gorelik, R. I. Shekhter, and M. Jonson, Phys. Rev. B 65, 165312 (2002). 
[18] M. Prada, G. Platero, and D. Pfannkuche, arXiv:1307.7576.

[19] D. V. Scheible, C. Weiss, J. P. Kotthaus, and R. H. Blick, Phys. Rev. Lett. 93, 186801 (2004).

[20] MATCONT and AUTO-07p are numerical continuation software applications, freely available at http://
www.matcont.UGent.be and http://indy.cs.concordia.ca/ auto/.

[21] D. V. Scheible, C. Weiss, and R. H. Blick, J. Appl. Phys. 96, 1757 (2004).

[22] C. Weiss and W. Zwerger, Europhys. Lett. 47, 97 (1999). 